\title{
Introduction to the Minitrack Software Sustainability: Strategies for Long-Lasting and Usable Software
}

\author{
Maytal Dahan \\ Texas Advanced Computing Center, \\ The University of Texas at Austin \\ maytal@tacc.utexas.edu
}

\author{
Joe Stubbs \\ Texas Advanced Computing Center, \\ The University of Texas at Austin \\ jstubbs@tacc.utexas.edu
}

\author{
Sandra Gesing \\ Center for Research Computing \\ University of Notre Dame \\ sandra.gesing@nd.edu
}

\begin{abstract}
The focus on software usability, long-lasting and reproducible software is a timely one that spans various domains of science and significant investment of research funding across the US, Europe, U.K, and elsewhere. The three concepts - usability, sustainability and reproducibility are interconnected with each other and cover a wide range of application areas. They affect all layers of the software process from enabling reproducing experiments via an easy user interface to using containerization for application portability. This minitrack focused on the broad spectrum of submissions that deal with complex scenarios such as containerization, strategies for long-lasting software, usability and user interface issues, handling data curation and provenance and more.
\end{abstract}

\section{Introduction}

The three concepts usability, sustainability and reproducibility are interconnected with each other and cover a wide range of application areas. They affect all layers of the software process - from enabling reproducing experiments via an easy user interface to using containerization for application portability. Such concepts are also relevant in the building of Science Gateways (also known as virtual laboratories or virtual research environments), which by definition serve communities with end-to-end solutions tailored specifically to their needs. Software survivability involves a wide scope that can potentially include the following topics:

- Web-based solutions (web sites, science gateways, virtual labs, etc.)

- Application Programming Interfaces (APIs)

- Computational and Data-Intensive Workflows

- Novel approaches in containerization
- Sustainability practices in software development

- System architectures for testing and continuous integration

- Emerging best practices in Machine Learning software

- Best practices and Key Success Factors for usability, survivability and reproducibility

This minitrack, Software Sustainability: Strategies for Long-Lasting and Usable Software, introduces the wide variety of accepted papers to HICSS-55. It focused on the broad spectrum of submissions that deal with complex scenarios such as containerization, strategies for long-lasting software, usability and user interface issues, handling data curation and provenance and more.

\section{Accepted Papers}

The minitrack received six papers with an excellent breadth of topics from software engineering with microservices to research software engineers and their roles to social responsibility to the role of the quantum computing field in sustainability. We accepted two papers to this minitrack that introduce the following topics:

- Attribution and awareness of sustainability

- Sustainability in microservices

One paper selected for this minitrack, "Software Engineers in Transition: Self-Role Attribution and Awareness for Sustainability" presents the role of software engineers in regard to self-awareness and sustainability. The paper dives into the perspective of software engineering being a socio-technical activity that involves fulfilling one's role as part of a team. Sustainability, which this paper interprets as the ability of a system to endure, is becoming part of the process and thus the responsibility of software engineers. The 
finding of this study is based on 13 interviews which shines the spotlight on the role of the software engineer in self-attribution and their awareness for sustainability. The paper attempts to figure out how they perceive their own role and to which extent they implement the topic of sustainability in their daily work. By visualizing these two sides, it is possible to debate changes and their possible paths to benefit the software engineering process including sustainability design.

Lastly the authors of the paper, "Public Safety Secretariat of Mato Grosso Microservice Environment" presents the microservice environment of the Public Safety Secretariat of Mato Grosso (SESP-MT) which was conceived to allow a migration process from SESP-MT monoliths and to absorb new organizational agile requirements. The project goes into the design of 22 containers with several tools and services that were assembled, tested and deployed in the SESP-MT environment integrated with the DevOps pipeline. The paper narrates complete experience and describes the motivations for choosing microservice architecture pattern, describes a base model, security and deployment pattern. The paper's contribution lies in detailing the successful environment implementation that can help other organizations in their microservices design.

\section{Conclusion}

These papers show a range of applications and impact of software sustainability in production and research software. They cover crucial aspects such as reproducibility and cultural approaches. We hope you will join us for interesting presentations and lively discussions on software sustainability, reproducibility, challenges, and solutions for our evolving landscape.

We aim at continuing with this minitrack in the future at HICSS and encourage authors to contribute their research and viewpoints on software sustainability with its many facets and areas. 